\title{
Saliendo de la zona de confort para mejorar las competencias y empleabilidad del alumnado
}

Begoña Cabanés-Cacho ${ }^{a}$, Esperanza Reina López ${ }^{\mathrm{a}}$, Guillermo Quilez Calavia ${ }^{\mathrm{b}}$, Antonio Montañés Gómez $^{\mathrm{b}}$, Franco Rutkevicius Remondinic ${ }^{\mathfrak{c}}$ Jaime Ramos Sevillano ${ }^{\mathfrak{c}}$, Miguel Magreñán Ovejas ${ }^{\mathrm{c}}$, Eneko Marcos Martínez ${ }^{\mathrm{c}}$

a Departamento de Dirección y Organización de Empresas, Facultad de Economía y Empresa, Universidad Zaragoza,

${ }^{\mathrm{b}}$ Exalumnos de la Facultad de Economía y Empresa, Universidad de Zaragoza

c Alumnos de la Facultad de Economía y Empresa, Universidad de Zaragoza

\section{\$EWWFW}

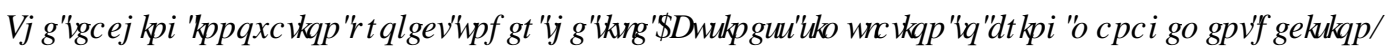

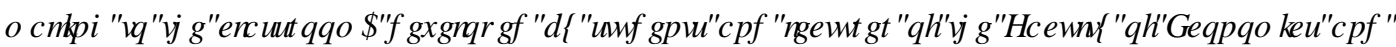
\%XYQHWURI UKH

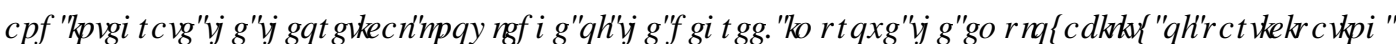

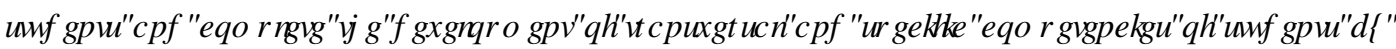
ODIQJ IKKHUFRP IRUWHRQH

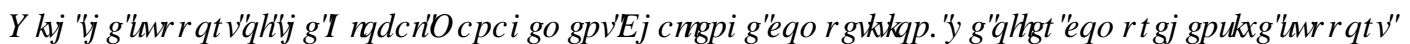

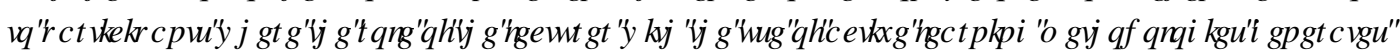

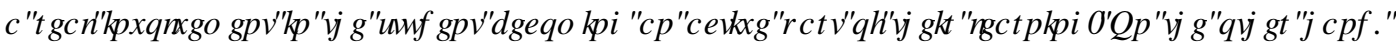

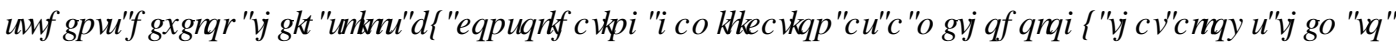

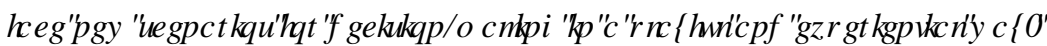

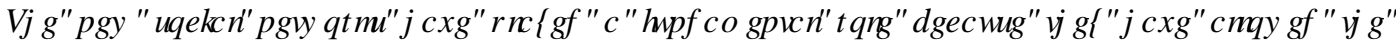

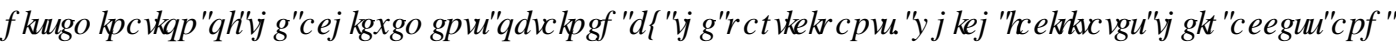
YIMEIOWIQUKHZ RLCHRIZZRLNI

[

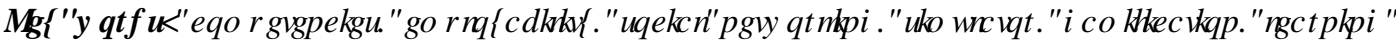
SLRFHMIODLQIQIISURFHM

(]

\section{HXP HQ}

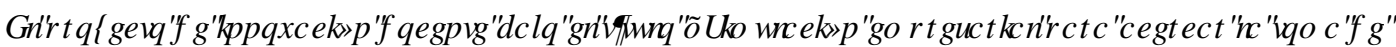

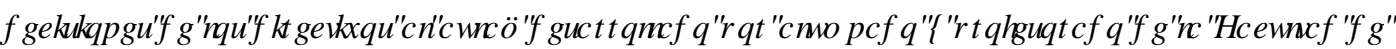
( FRQRP tDD I ( P SUMD GH QD 8 QIYHUGDG GH =DDJ R]DD KD SHUH XIGR GHGH WD FUHFIYQ WHW

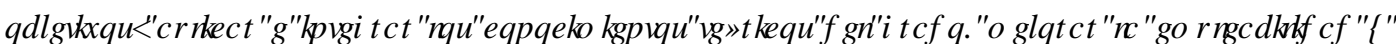

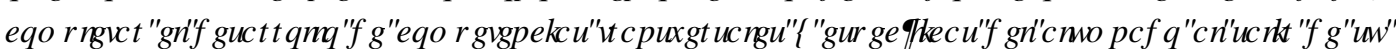
JRQDIGHFRQIRUW

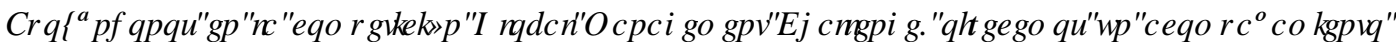

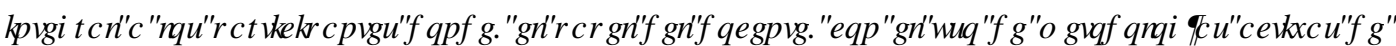

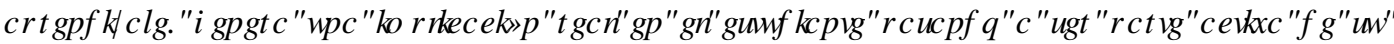

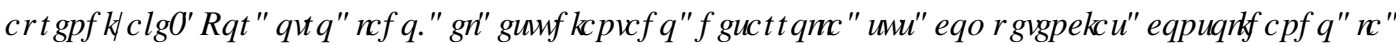




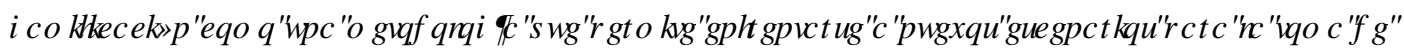
GFLMRQHKGHXQDIRUP DOGFDI पYIYHQFLD

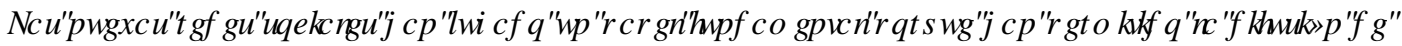

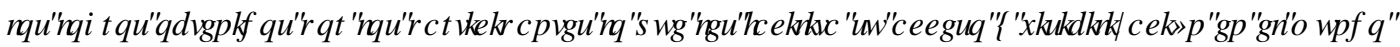
QDERLOD

प

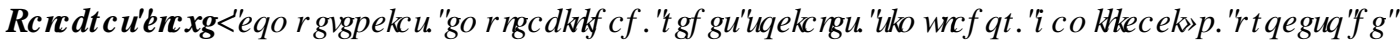 DSUHQG]DNAII}

प

\section{Introducción}

¿Y si nos hubieran encargado una misión de capital importancia? Imaginemos que tuviéramos una misión que cumplir, el solo encargo, el breve enunciado parece en sí mismo retador.

¿Y si esa misión la tienen que desempeñar los docentes?

¿Y si... para entender la esencia de la referida misión, nos remontáramos a la etimología por si puede ofrecernos alguna pista válida? Etimológicamente, «docente» es el participio de presente del verbo latino DOCĚRE 'enseñar', una antigua formación causativa que significaba, literalmente, 'hacer que alguien aprenda ${ }^{1}$, enseñar.

¿Y si la misión fuera realizar algún proceso / método alternativo de aprendizaje a los tradicionales que ayudara a nuestros estudiantes a prepararse para su vida "futura"?

¿Y si implicáramos a parte del estudiantado?

La misión que anticipábamos podía ser retadora, ha pasado ciertamente a serlo y, para darle respuesta, podríamos iniciar distintas vías.

Desde el punto de vista más racional, sería lógico pensar que, para predecir las competencias necesarias para un desempeño idóneo en el futuro, debemos partir del entorno actual y los cambios de tendencias que se anticipan y en muchos casos, adivinan.

El entorno actual está caracterizado por una constante, el cambio, y en diversos ámbitos: tecnológicos, social, culturales, competidores, negocios.

Siguiendo con nuestra secuencia lógica y dada la imposibilidad de tener la certeza en cada momento de cuál va a ser la tendencia, el avance, el siguiente cambio, nuestros estudiantes, tendrán que desarrollar de manera muy importante las denominadas habilidades blandas (VILWNC), entre las que podemos citar sin ambición de exhaustividad: capacidad de liderazgo, trabajo en equipo, cortesía, orientación al cambio, flexibilidad, empatía, asertividad, pensamiento crítico, poder de negociación , persuasión, capacidad de comunicación y resiliencia.

¿Y si... encontráramos un proyecto, una fórmula complementaria en tiempo, contenido y objetivos a la más estrictamente curricular y "obligatoria”, que posibilitará reforzar los conocimientos más teóricos adquiridos de forma "académicamente tradicional" de manera complementaria a otras competencias que hemos definido como claves para el presente y ese hipotético futuro?

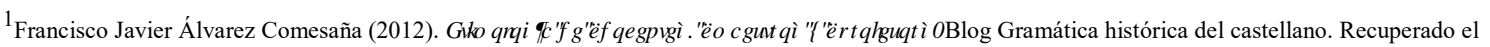
15 de marzo de 2021, de https://www.delcastellano.com/etimologia-docente-maestro-profesor/
} 
¿Y si a todo lo verbalizado anteriormente le podemos dar respuesta con un proyecto participativo y voluntario?

La competición de Global Management Challenge (en adelante, GMC) se ha convertido en la ayuda que necesitábamos para completar la formación. Esta competición invita a todos los estudiantes universitarios españoles a aprender a dirigir empresas en un entorno competitivo similar al de una empresa real. Llevando a los participantes a verse inmersos en un mundo construido para la ocasión, muy similar al real, donde las situaciones, los objetivos y las decisiones sean un verdadero entrenamiento para un futuro laboral cercano.

Apoyándonos en Ayala $H M D O$ (2020) y su opinión sobre las oportunidades pedagógicas que otorgan los entornos 3D, y extrapolándolas al uso de simuladores empresariales, su utilización hace que los estudiantes desarrollen habilidades transversales y no disciplinares, no limitándose al conocimiento teórico implantado en cursos tradicionales. Esta combinación de métodos de enseñanza permite que florezcan capacidades y habilidades intercurriculares, convirtiéndolo en un proceso más intrínsecamente motivador para algunos estudiantes (Abbott HMDO2017).

En este proyecto en el que estamos embarcados desde 2019, el objetivo principal es tutorizar y acompañar al alumnado que así lo solicite, de todos los grados de la Facultad de Economía y Empresa de la Universidad de Zaragoza, a lo largo de las diferentes fases de la competición GMC. En la última edición en la que hemos participado durante el curso 2020-21, en la primera ronda se enfrentaban 30 equipos en 4 mercados (grupos) de los cuales tutorizamos y acompañamos a 9 equipos (34 estudiantes).

Como comenta Cuevas $H W D O(2021)$ el abordaje del desarrollo de las competencias desde una perspectiva más lúdica y vivencial supone una redefinición de los procesos formativos y la experiencia de aprendizaje discente, a la vez que contribuye a la creación de nuevos escenarios donde poder diseñarlos e implementarlos. Estas cuestiones han permitido consolidar la gamificación como una metodología habitual para el desarrollo de acciones formativas en las diferentes etapas.

No podemos dejar a la tecnología sin ocupar el lugar que debe, ya que ha jugado un papel fundamental, por un lado, durante la competición, facilitando el trabajo colaborativo en la distancia a los componentes de cada grupo participante, y por otro, al terminar, permiten la difusión de los resultados y de los logros conseguidos por los estudiantes de manera segura e inmediata, que les facilitará su acceso al mundo laboral. A su vez, nos sirven como reclamo de participación para futuros estudiantes.

Todo esto permite al estudiante tomar las riendas en su formación y salir de la zona de confort. Tal y como comenta Hidalgo (2020) es imprescindible que los docentes desarrollen escenarios alternativos en la Zona de Desarrollo Próximo (ZDP) y que con metodologías activas impulsen el desarrollo potencial del alumnado.

Así, podríamos decir que nuestro proyecto, se asienta sobre 5 pilares:

- El uso de la gamificación como herramienta indiscutible generadora de implicación. En la competición el mercado está generado por el simulador GMC certificado por la European Foundation for Management Development (EFMD) en Cursos de Educación Online (EOCCS) ${ }^{2}$.

- El aprendizaje social o colaborativo, en el que, además gracias a las redes posibilita que los estudiantes compartan y aprendan de manera recíproca contribuyendo el aprendizaje en grupo y creando espacios de trabajo en donde los estudiantes que se encuentren lejos puedan reunirse o conectarse para el intercambio experiencial y cognitivo (Stefan, 2012)

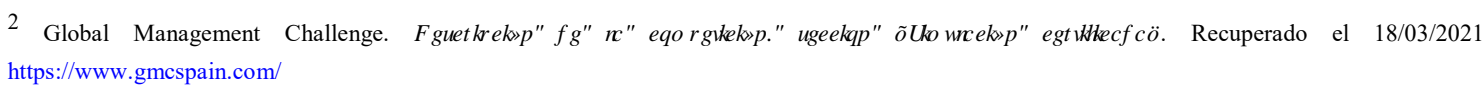

(c)) BY-NC-ND 2021, Universitat Politècnica de València

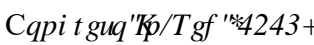


- El carácter voluntario, que genera una implicación, que va aumentando a medida que avanza la competición.

- $\quad$ El hecho de que el grupo de tutores está formado por profesores y por estudiantes que han ganado la fase autonómica y han llegado a la competición a nivel nacional. Ambos hacen de coach para acompañar y ayudar en las reflexiones para la toma de decisiones de los distintos equipos tutorizados. Los profesores a través de preguntas y reflexiones sobre diferentes aspectos teóricos y cómo ponerlos en práctica, y los estudiantes aportan su experiencia y know-how con el simulador. Ya que hay que tener en cuenta que los diferentes equipos están compitiendo entre ellos, con lo cual los tutores no pueden favorecer a ningún equipo.

- El "plus" en el curriculum de los estudiantes participantes, debido a la transcendencia y difusión en las redes sociales y diferentes medios de comunicación.

¿Y si tenemos la certeza de que hemos encontrado una respuesta al reto?

\section{Objetivos}

El principal objetivo por el cual desarrollamos este proyecto de innovación docente es ayudar a los estudiantes en su proceso de aprendizaje en su recta final hacia su desarrollo profesional en el mundo real.

En este artículo, vamos a analizar como su participación en una competición basada en la simulación junto a un acompañamiento (tutorías siguiendo la metodología coaching) ha sido un buen escenario para que los estudiantes salgan de su zona de confort y desarrollen habilidades enfocadas a su empleabilidad.

Los objetivos específicos son:

1. Aplicar e integrar los conocimientos teóricos del grado.

2. Mejorar la empleabilidad del alumnado a través de la diferenciación y la visibilización tras su participación en GMC.

3. Completar el desarrollo de competencias transversales y específicas del alumnado al salir de su zona de confort con la creación de una Zona de Desarrollo Próximo (ZDP).

Para valorar si se alcanzan los objetivos se realiza a los estudiantes una encuesta basada en la competición y analizaremos si han realizado publicaciones sobre su participación en LinkedIn.

\section{Desarrollo de la innovación}

Desde el Proyecto de Innovación Docente "Simulación empresarial para acercar la toma de decisiones de los directivos al aula (PIIDUZ_19_451)" se planteó la posibilidad al estudiantado de la Facultad de Economía y Empresa participar en el reto GMC.

GMC es una competición de estrategia y gestión empresarial en la que participan estudiantes universitarios. Los participantes forman equipos de 2 a 4 miembros que son ubicados en grupos/mercados de hasta ocho equipos, en función de la ronda de competición. Al inicio de cada ronda los equipos reciben una empresa virtual completamente igual que deben gestionar (GMC, 2021)

Se ofreció al alumnado que decidió participar un equipo de tutores, asignado de manera aleatoria si así lo solicitaban los equipos. Los tres equipos de tutores, formados cada uno de ellos por un/a profesor/a de la Facultad y un integrante de cada uno de los equipos campeones de Aragón de las ediciones pasadas 2020 (Equipo JEM) y 2019 (Equipo GAF) que acompañaron a los equipos solicitantes.

Se usó un planteamiento basado en el coaching educativo, dicho con otras palabras, acompañarlos en el desarrollo de sus competencias, así como en el desarrollo integral del alumno desde la motivación, (Ávila 
$H M D O, 2020)$ buscando los mejores resultados sin decirles directamente las respuestas, pero creando un entorno apropiado a base de preguntas para que ellos saquen sus propias conclusiones.

De cara a la futura incorporación al mercado laboral del estudiante, participar en una competición como GMC, justifica diferentes capacidades y habilidades que adquiere el participante. Es una forma de diferenciación, creando valor, mediante unas VRIWWNCladquiridas y desarrolladas durante todo el concurso que cada vez son más demandadas en el mundo de la empresa.

El autoaprendizaje al que se somete al alumnado es vital, desde un escenario virtual, aprende a desarrollar las herramientas necesarias para el mundo actual. Esta competición exige a los participantes analizar la información desde el pensamiento crítico, trabajar en equipo para tomar las mejores decisiones en base a la estrategia que hayan definido a través de la negociación, siendo clave anticiparse a las decisiones que tomen el resto de equipos y teniendo una clara orientación a la gestión de la empresa y su viabilidad.

El alumnado se sumerge en un aprendizaje significativo gracias a su participación en entornos virtuales, basados en las tecnologías de la información, las redes sociales y la ludificación que nos permiten lograr procesos pedagógicos complejos como explican Ly $\square$ HWDO, 2017 y Ayala HWDO, 2020. En nuestro caso, la competición permite poner en práctica todos los conocimientos teóricos que ha ido adquiriendo durante su paso por la universidad. De esta forma, afianzan e interiorizan conceptos completando así un aprendizaje global de forma transversal.

Otro de los aspectos clave es el simulador, por un lado, saca al alumnado de su zona de confort al tener que gestionar una empresa como actividad extracurricular. Por otro, permite a los tutores desarrollar una nueva metodología, coaching, que hace hincapié en la reflexión y pensamiento crítico. Y, por último, el autoaprendizaje, imprescindible para justificar el desarrollo de sus competencias en una futura entrevista de trabajo.

La competición se desarrolla en diferentes fases. La primera fase se celebra durante los meses de noviembre y diciembre de 2020 donde 30 equipos repartidos en 4 mercados, tienen que dirigir una empresa virtual durante 5 semanas equivalentes a 5 trimestres, tomando en cada una de ellas 77 decisiones sobre producción, finanzas, marketing y recursos humanos. Los dos mejores equipos de cada mercado se clasifican para la Final Autonómica donde competirán 8 equipos en un solo mercado. El equipo que quede en primera posición pasara a la fase nacional.

Durante la primera fase los equipos solicitan tutorías, estas se desarrollan a través de plataformas de videollamada como Meet. Tras las tutorías los equipos tomaban sus decisiones y las incorporaban al simulador. De los 9 equipos tutorizados solo 6 podían clasificarse, pero solo completaron el proceso 6 equipos de los cuales se clasificaron 4 para la final autonómica. Dicha final se desarrollaba en una única jornada donde tienen que tomar 5 decisiones, además de estar incomunicados y no poder recibir tutorías. Con lo cual, nuestra labor ha sido previa a ese día para ayudarles a reflexionar y plantear posibles escenarios para que vayan desarrollando su capacidad de toma de decisiones, reparto de tareas y gestión del liderazgo.

Todos los equipos reciben la misma información sobre la empresa que van a gestionar donde se muestran datos sobre los cinco trimestres previos, como son el balance, cuenta de pérdidas y ganancias, numero de máquinas, cantidad de empleados, tipo de productos, mercados donde los venden, ... Igual que si tuvieran que enfrentarse con una empresa real en el mercado. Las decisiones se envían a través de una plataforma, y los resultados de su decisión se muestran en una Excel. La empresa puede fabricar hasta tres productos y puede vender en tres mercados, todo ello esta explicado en un manual. En base al análisis de esta información cada equipo tiene que definir su estrategia, que abanderará su comportamiento durante los cinco trimestres que la gestiona. En cada trimestre tienen que tomar las siguientes decisiones: 
- Producción: se determina la compra de materia prima o si se subcontrata parte del proceso, las horas de mantenimiento de las máquinas, numero de turnos para producir, y las cantidades a entregar de los tres productos en los tres mercados.

- Finanzas: Ampliación de capital, distribución de dividendos, decisiones de inversión o desinversión en maquinaria, ampliación de fábrica, solicitud de préstamos o gestión de inversiones.

- Marketing: Investigación y desarrollo en cada uno de los tres productos, precios de comercialización, agentes y distribuidores en cada uno de los mercados, así como negociación de sus comisiones, mejoras a aplicar en cada uno de los productos, y compra de información del mercado.

- Recursos humanos: número de operarios, su salario y gastos de formación tanto para operarios como para staff.

Las decisiones a tomar en la empresa virtual son similares a las que se toman en cualquier empresa manufacturera real.

\section{Resultados}

En ediciones anteriores hemos visto como ha sido la repercusión para el alumnado que ha llegado a la final autonómica, gracias a difusión en redes sociales, de la organización GMC, Facultad de Economía y Empresa, medios de comunicación, y las publicaciones realizadas por los tutores y los propios estudiantes que han servido de llamamiento a las empresas que buscaban candidatos para realizar prácticas, becas y contratos laborales.

Vamos a analizar los resultados en base a los objetivos planteados en este artículo para ello obtendremos la información de la encuesta que realizaron los estudiantes tras su participación y la repercusión de las publicaciones realizadas en LinkedIn por los mismos.

La encuesta fue respondida por 17 personas de las 30 que inicialmente se incorporaron al proyecto.

En el cuestionario, encontramos varias secciones. Una de ellas tiene que ser respondida con una valoración del 1 al 5, siendo 1 completamente en desacuerdo y 5 completamente de acuerdo. Otra sección tiene que ser respondida con "Sí", "No" o "Tal vez". Estas son útiles, ya que podemos obtener gráficos con las mismas. El último tipo de pregunta es de opción múltiple, donde el alumno puede elegir varias respuestas.

Respecto al primer objetivo "aplicar e integrar los conocimientos teóricos del grado" a continuación en la Tabla 1 se adjuntan las afirmaciones del cuestionario directamente relacionadas con el mismo junto con la media obtenida.

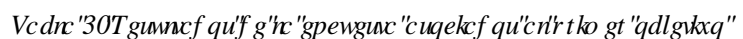

\begin{tabular}{|l|c|}
\hline \multicolumn{1}{|c|}{ Afirmaciones } & $\begin{array}{c}\text { Puntuacion } \\
\text { entre 1 y 5 }\end{array}$ \\
\hline Me ha ayudado a entender el funcionamiento de la empresa. & 4,47 \\
\hline Actividades como esta permiten integrar mejor los conocimientos. & 4,53 \\
\hline La actividad ha sido relevante para mi formación. & 4,24 \\
\hline
\end{tabular}

Fuente: Elaboración propia

Los participantes valoraron con un 4,47 de media el hecho de participar en este simulador puesto que les ha ayudado a entender el funcionamiento de una empresa. Consideran que ha sido de gran ayuda puesto 
que en la competición toman decisiones sobre las principales áreas de una organización. De esa forma, comprueban como las decisiones de cada departamento afectan al resto.

Los participantes opinan, con un 4,53 de media, que este tipo de actividades permiten integrar mejor los conocimientos. Les ha permitido de una forma más dinámica y competitiva poner en práctica conocimientos adquiridos en su formación académica.

Por último, respondieron con una media de 4,24 que la actividad había sido relevante para su formación. Por lo tanto, piensan que los conocimientos adquiridos con el simulador les son útiles y complementarios a lo aprendido en la facultad.

El segundo objetivo del proyecto es "mejorar la empleabilidad del alumnado a través de la diferenciación y la visibilización tras su participación en GMC". Para analizar este objetivo lo hemos dividido en dos bloques, el primero la diferenciación y el segundo la visibilización por redes sociales y blogs.

Para analizar la diferenciación, ya el hecho de participar en una actividad extracurricular marca una diferencia en los estudiantes involucrados, por un lado, se trata de un simulador con gran reputación a nivel nacional e internacional, y, por otro lado, permite desarrollar habilidades de gestión, trabajo en equipo y manejar la presión en la toma de decisiones, plus que marca la diferencia a la hora de realizar una entrevista o de justificar sus competencias. A continuación, en la Tabla 2 se adjuntan las afirmaciones del cuestionario directamente relacionadas con el mismo, junto con la media obtenida.

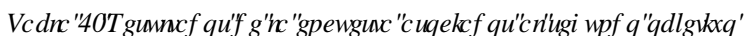

\begin{tabular}{|l|c|}
\multicolumn{1}{|c|}{ Afirmaciones } & $\begin{array}{c}\text { Puntuacion } \\
\text { entre 1 y 5 }\end{array}$ \\
\hline $\begin{array}{l}\text { He aprendido y desarrollado habilidades que me ayudaran a mi futura } \\
\text { empleabilidad. }\end{array}$ & 4,12 \\
\hline $\begin{array}{l}\text { Me ha permitido mejorar mis habilidades profesionales (resolución de } \\
\text { problemas, organización, análisis y procesamiento de información,...). }\end{array}$ & 4,18 \\
\hline
\end{tabular}

Fuente: Elaboración propia

Ambas afirmaciones obtuvieron una media considerable ( 4,12 y 4,18 sobre 5 respectivamente), por lo que podemos afirmar que el alumnado considera que ha mejorado sus habilidades necesarias para su futura vida laboral.

Con respecto a la idea de visualización de cara a la empleabilidad, lo hemos analizado desde dos puntos de vista, por un lado, como ellos creían que les iba afectar su participación y, por otro lado, analizando directamente las reacciones (comentarios y recomendaciones) en sus cuentas de LinkedIn.

En el primer análisis a través de la encuesta, todos ellos afirman que la participación en esta competición "ayudará" a su futura empleabilidad, respondiendo "Sí" con un 52,9\% de los votos y un "Tal vez" con un $47,1 \%$, no habiendo ninguna respuesta negativa. Es decir, todos consideran a priori que su participación va a ser beneficiosa para su futuro.

En segundo lugar, analizando las publicaciones en LinkedIn realizadas por el estudiantado que participó en la final de Aragón, 8 de los 15 participantes realizaron una publicación. Se observó una media de 6 comentarios y 19 recomendaciones, que relacionándolo con la media de contactos por participante (118) supone un $5 \%$ en comentarios y $16 \%$ recomendaciones. Cantidades relativamente altas, puesto que la 
mayoría acaba de darse de alta en esta red social orientada al uso profesional, empresarial y empleo. Siendo todos ellos estudiantes, aunque algunos han comenzado a tener experiencia profesional.

No podemos dejar de mencionar la repercusión que ha tenido su participación al aparecer sus nombres e imágenes en los blogs y redes sociales de la competición GMC y de la Facultad de Economía y Empresa (Universidad de Zaragoza).

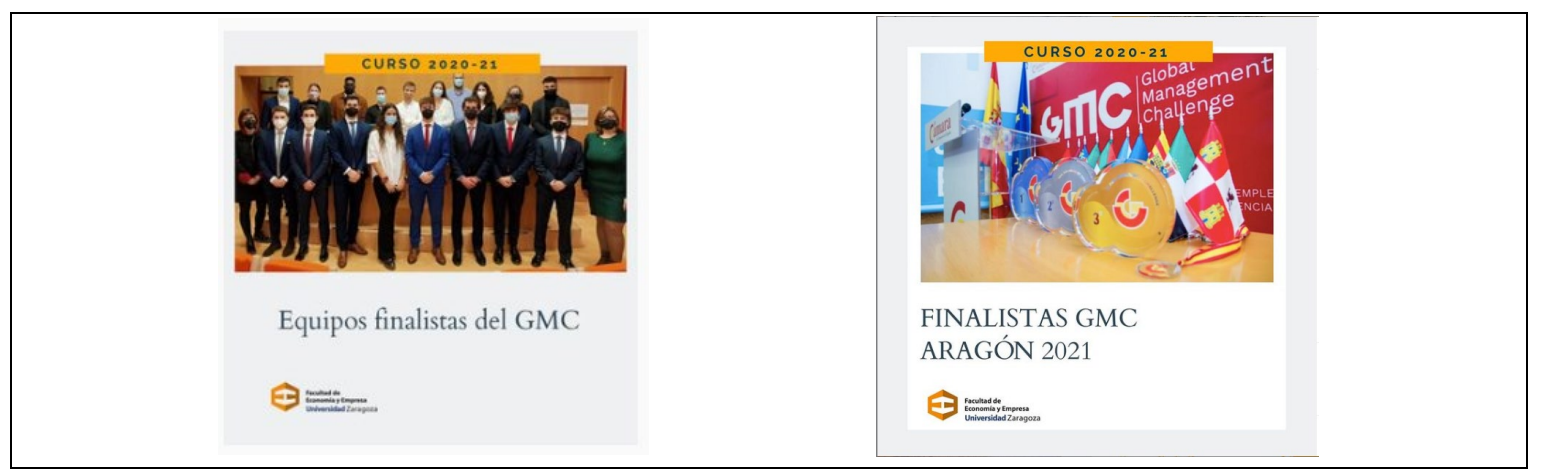

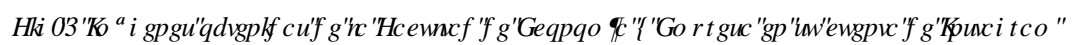

प

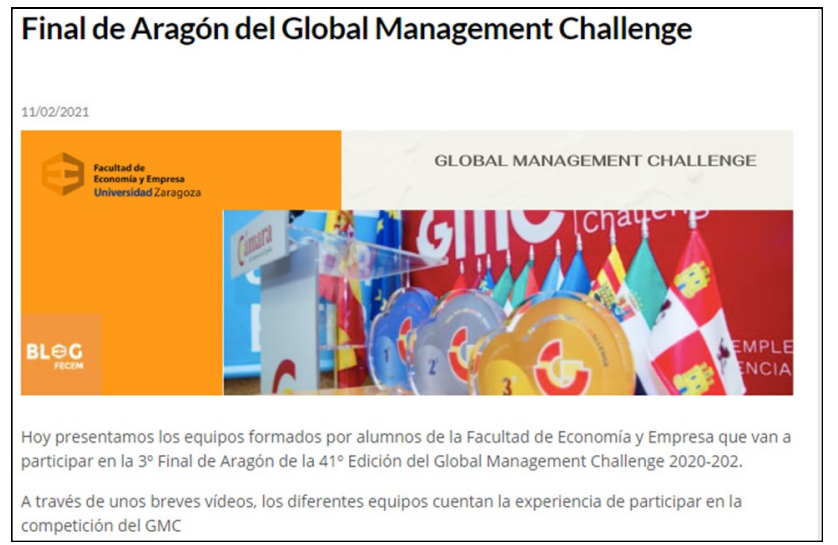

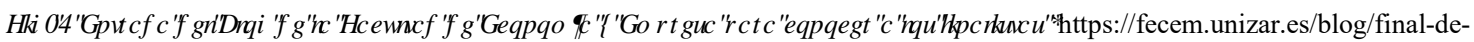
aragon-del-global-management-challenge[ए

El tercer y último objetivo de este proyecto es completar el desarrollo de competencias transversales y específicas del alumnado al salir de su zona de confort con la creación de una Zona de Desarrollo Próximo (ZDP).

Basándonos en las respuestas de los participantes en el cuestionario que adjuntamos en la Tabla 3, analizadas previamente para el primer y segundo objetivo, consideramos que los estudiantes han salido de su zona de confort al participar en una actividad extracurricular, suponiendo para ellos una exigencia autoimpuesta (aproximadamente con una dedicación semanal de 3 horas en la primera parte de la competición). Además, se trata de una zona de desarrollo próximo ya que se mantiene en el entorno universitario y con tutores cercanos. 
7DEDD П5 HXQDCRVGHDHQFXHWDVRFIDRVDOMHFHURENAMR

\begin{tabular}{|l|c|}
\hline \multicolumn{1}{|c|}{ Afirmaciones } & $\begin{array}{c}\text { Puntuacion } \\
\text { entre 1 y 5 }\end{array}$ \\
\hline Actividades como esta permiten integrar mejor los conocimientos. & 4,53 \\
\hline $\begin{array}{l}\text { Me ha permitido mejorar mis habilidades profesionales (resolución de } \\
\text { problemas, organización, análisis y procesamiento de información,...). }\end{array}$ & 4,18 \\
\hline
\end{tabular}

Fuente: Elaboración propia

Basándonos en estas dos respuestas, podemos afirmar que los alumnos completaron su desarrollo de competencias transversales y específicas.

Nos parece relevante exponer la siguiente información obtenida del cuestionario:

- El 95\% participaría de nuevo en la siguiente edición.

- $\quad$ El 100\% de los encuestados recomiendan la competición.

\section{Conclusiones}

Apoyándonos en el programa de simulación empresarial utilizado en la competición GMC hemos dado al proceso de aprendizaje, un enfoque diferente al convencional, que nos ha permitido hacer crecer a los estudiantes, retándolos a reflexionar sobre sus decisiones, aplicar lo que ya conocen, y a sentirse mucho más cómodos fuera de su zona de confort.

Para comprobar si hemos cumplido los objetivos planteados en el proyecto se ha realizado una encuesta a los participantes comentada en el apartado anterior de Resultados. Podemos afirmar que hemos cumplido los tres objetivos, ya que este nuevo escenario dentro del entorno universitario, pero fuera del aula, ha permitido al alumnado adaptarse. Por un lado, a las nuevas tecnologías a través del simulador y herramientas de comunicación online, necesarias a causa de la pandemia. Y, por otro lado, han aplicado los conocimientos teóricos en este entorno próximo y, al tratarse de una actividad voluntaria, les ha permitido diferenciarse de sus "rivales" de cara a futuras entrevistas, al poder justificar el desarrollo de sus competencias transversales tales como la toma de decisiones, análisis de información y pensamiento crítico, entre otras.

Podemos destacar el proceso de apertura a las redes sociales profesionales por parte del alumnado que les ha permitido promover su visibilidad a la vez que supone salir de su zona de confort. Ya que han aprendido a "venderse" y poner en valor sus competencias a la hora de realizar sus publicaciones o realizar comentarios en las publicaciones de la facultad y de la propia competición.

En la actualidad, el profesorado de este proyecto pertenece al área de organización de empresa que está presente en diferentes áreas de conocimiento como economía, ingeniería, periodismo, .... Y, aunque el proyecto hasta la fecha solo se ha llevado a cabo con estudiantes del área de economía, el hecho de que la competición GMC esté abierta a todos los estudiantes universitarios, nos hace plantearnos la posibilidad de una futura participación del profesorado de otras facultades de nuestra universidad con el fin de hacer un trabajo más transversal. Esto nos permite afirmar que es una actividad extrapolable a un contexto más amplio puesto que facilita el desarrollo de competencias transversales demandadas en el mercado laboral e incluso favorecer el emprendimiento, perdiendo el miedo a la toma de decisiones. 
Se va a solicitar nuevamente el proyecto de innovación docente aprovechando la experiencia para una mejora continua. Además, es momento de afianzar la competición en Aragón, siendo su cuarta edición y la $42^{\circ}$ a nivel nacional.

Por último, desde el equipo de tutores nos sentimos muy orgullosos de haber acompañado a estos participantes y la evolución que han desarrollado los mismos. Siendo destacable que el $100 \%$ de los encuestados recomiendan participar en la competición.

\section{Agradecimientos}

A la Universidad de Zaragoza por la concesión del Proyecto de Innovación Docente titulado "Simulación empresarial para acercar la toma de decisiones de los directivos al aula" (PIIDUZ_19_451).

\section{Referencias}

Abbott, D., Jeffrey, S., Gouseti, A., Burden, K., \& Maxwell, M. (2017). "Development of cross-curricular key skills using a 3D immersive learning environment in schools" en \&RP P XQIFDMRQVIQ \&RP SXWUDDQG ,QRRP DURQ6FHQFH प0०प60-74. doi: https://doi.org/10.1007/978-3-319-60633-0 6

Ávila Guerrero, F. M., Gutiérrez-Tobar, E. \& Vargas- Vargas, S.A. (2020). "Lineamientos de coaching que puede aplicar el docente como estrategia para potenciar el aprendizaje”. , Q9HWWLXP, UHШVol. 14-1, pp. 12- 26.

Ayala, R., Laurente, C., Escuza, C., Núñez, L., \& Díaz, J. (2020). "Mundos virtuales y el aprendizaje

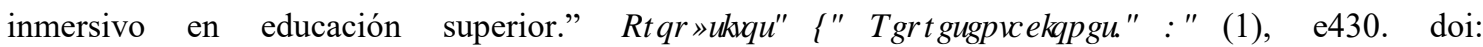
http://dx.doi.org/10.20511/pyr2020.v8n1.430

Cuevas Monzonís, N, Cívico Ariza, A, Gabarda Méndez, V y Colomo Magaña, E (2021). "Percepción del alumnado sobre la gamificación en la educación superior”. 5( ,' 2 \&5( \$山्(16), 1-12.

Global Management Challengue, ¿Qué es GMC?”. Available at: https://www.gmcspain.com/funcionamiento-global-management-challenge/ [Accedido: 15/03/2021]

Hidalgo, M.E. (2020). "Reflexiones acerca de la evaluación formativa en el contexto universitario". 5HLWD , QHAQDFIRQDOCHI3 HODJ RJ TDH, QQRYDFIy Q( GXFDUMD1 (1), 189-210. ISSN: 2745-0341 (En línea)

Ly, S., Saadé, R., \& Morin, D. (2017). "Immersive learning: Using a web-based learning tool in a phd

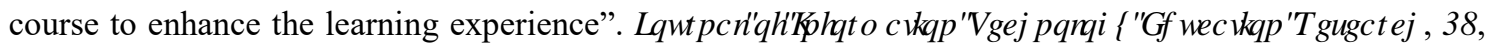
227-246. doi: https://doi.org/10.28945/3732

Stefan, L. (2012). "Immersive collaborative environments for teaching and learning traditional design".

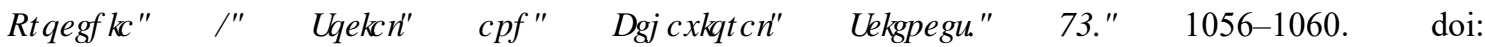
https://doi.org/10.1016/j.sbspro.2012.08.287 